\title{
Associations and prognostic implications of Eastern Cooperative Oncology Group performance status and tumoral LINE-1 methylation status in stage III colon cancer patients
}

Duo Chen ${ }^{1 \dagger}$, Xianyu Wen ${ }^{2 \dagger}$, Young Seok Song ${ }^{3}$, Ye-Young Rhee ${ }^{2}$, Tae Hun Lee ${ }^{3}$, Nam Yun Cho ${ }^{2}$, Sae-Won Han ${ }^{4}$, Tae-You Kim ${ }^{4}$ and Gyeong Hoon Kang ${ }^{2^{*}}$

\begin{abstract}
Background: Low methylation status of LINE-1 in tumors is associated with poor survival in patients with colon cancer. Eastern Cooperative Oncology Group performance status (ECOG-PS) is a method to assess the functional status of a patient. We retrospectively evaluated the relationship between ECOG-PS and LINE-1 methylation in colorectal cancers (CRCS) and their prognostic impact in CRC or colon cancer patients receiving adjuvant 5-fluorouracil/ leucovorin/oxaliplatin (FOLFOX).

Results: LINE-1 methylation and microsatellite instability were analyzed in stage III or high-risk stage II CRCs ( $n=336$ ). LINE-1 methylation levels were correlated with clinicopathological features, including PS and recurrence-free survival (RFS). The association between the tumoral LINE-1 methylation level and PS was observed (OR $=2.56, P<0.001$ ). Differences in LINE-1 methylation levels in cancer tissue between the PS 0 and 1 groups were significant in patients older than 60 years $(P=0.001)$, the overweight body mass index group $(P=0.005)$, and the stage III disease group $(P=0.008)$. Prognostic significances of LINE-1 methylation status or combined PS and LINE-1 methylation statuses were identified in stage III colon cancers, not in stage III and high-risk stage II CRCs. Low LINE-1 methylation status was closely associated with a shorter RFS time. The difference between PS(0)/LINE-1(high) and PS( $\geq 1$ )/LINE-1(low) was significant, which suggests that colon cancer patients with concurrent PS( $\geq 1) / L I N E-1$ (low) have a higher recurrence rate.
\end{abstract}

Conclusions: PS was associated with LINE-1 methylation in CRC tissue. LINE-1 methylation was associated with RFS in stage III colon cancer patients who were treated with adjuvant FOLFOX chemotherapy. Combined PS and LINE-1 methylation status might serve as a useful predictor of cancer recurrence.

Keywords: Adjuvant therapy, Colorectal cancer, ECOG, FOLFOX, LINE-1, Methylation, Prognosis

\footnotetext{
* Correspondence: ghkang@snu.ac.kr

${ }^{\dagger}$ Equal contributors

2Department of Pathology, Seoul National University College of Medicine,

Seoul, Korea

Full list of author information is available at the end of the article
} 


\section{Background}

Colorectal cancer $(\mathrm{CRC})$ is one of the most common cancers and a leading cause of cancer death globally [1]. Despite a declined CRC mortality in developed countries, the incidence and mortality are increasing in East Asian populations, including Korea [2, 3]. Adjuvant chemotherapy significantly decreases mortality in colon cancer, and 5-fluorouracil (5-FU), leucovorin, and oxaliplatin (FOLFOX) are the current standard of care for patients with stage III colon cancer after surgery $[4,5]$. FOLFOX is also frequently used to treat stage II colon cancer with high-risk features, such as T4 tumors or lymphovascular invasion.

CRC is a multifactorial disease which arises due to the accumulation of genetical and epigenetical alterations. Epigenetic changes are potential factors contributing to the carcinogenesis of colorectal cancer (CRC). Genomewide hypomethylation is an alternative mechanism for genomic instability, which facilitates tumor progression [6]. Methylation levels of repetitive transposable DNA elements are a useful surrogate marker for global genomic methylation status because repetitive DNA elements reside in intergenic or intronic regions of the genome at extremely high frequencies, and CpG sites located within repetitive DNA elements are usually methylated [7]. Long interspersed nucleotide element-1 (LINE-1) is a major constituent of repetitive transposable DNA elements, and it constitutes approximately $17 \%$ of the human genome [7, 8]. LINE-1 is usually methylated in normal cells, which maintains transcriptional inactivation and inhibits retrotransposition [9]. Hypomethylation of $L I N E-1$ is a common finding in various tissue types of human cancer, and recent meta-analysis results have shown that LINE-1 methylation levels are significantly lower in cancer tissues than in paired normal tissues, and especially in CRC and gastric cancer [10-17]. In some tissue types of human cancer, including CRC, tumoral LINE-1 hypomethylation is associated with a poor clinical outcome [14, 15, 18-21].

The Eastern Cooperative Oncology Group performance status (ECOG-PS) is a global assessment of a cancer patient's actual level of function and self-care ability $[22,23]$. ECOG-PS is strongly associated with prognosis in various tissue types of cancer, including CRC [24-31]. Poor PS (PS score $\geq 2$ ) is a strong predictor of poor prognosis in patients with metastatic CRC and tends to be associated with poor prognosis in patients with nonmetastatic CRC [32, 33]. Although increasing stages of tumor depth or nodal metastasis tend to be accompanied by functional declines of the patients, little is known regarding the relationship between PS and cancer biology, including molecular phenotypes. A couple of recent studies have shown that a decrease in tumoral DNA methylation content leads to endogenous retrovirus activation and subsequent overexpression of interferonpathway genes [34, 35]. Proinflammatory signals from tumor cells or aberrant host response to tumor presence might affect PS of the cancer patient [36]. Thus, it can be speculated that PS might play a confounding role in the effect of tumoral LINE-1 methylation status as a predictor of recurrence. Recently, Lou et al.'s study has demonstrated that low LINE-1 methylation status is an independent risk factor for recurrence in stage III colon cancer patients treated with adjuvant FOLFOX [37]. However, the study of Lou et al. did not consider PS as a potential predictor of recurrence. In this study, we analyzed adjuvant FOLFOX-treated stage III or high-risk stage II CRCs for their methylation levels of LINE-1 using pyrosequencing methylation assay and correlated tumoral LINE-1 methylation status with multiple clinicopathological parameters, including PS and recurrencefree survival of the patient.

\section{Results}

\section{Distribution of clinicopathological parameters in different PS groups}

A total of 336 patients were included. Clinicopathological parameters of the patients are described in Table 1. PS $\geq 1$ was present in 170 (50.5\%) patients (169 patients with PS 1 and one patient with PS 2). The majority of patients were men $(n=211,62.8 \%)$, and the average age was 58.9 years (median 60, range 29-78 years). The tumor location was cecum in 9 patients, ascending colon in 75 , transverse in 21 , descending in 22 , sigmoid in 189 , and rectum in 20 , including 104 right colon carcinomas and 212 left colon carcinomas. $T$ stages $1 / 2 / 3 / 4$ were $8 / 30 / 255 / 43$ in patients, respectively, and $N$ stages $0 / 1 / 2$ were $49 / 203 / 84$ in patients, respectively. Forty-nine patients had high-risk stage II disease (IIA, 33; IIB, 12; IIC, 4), and 287 patients had stage III disease (IIIA, 31; IIIB, 195; IIIC, 61). A total of 165 patients received FOLFOX-4, and 171 patients received modified FOLFOX-6. KRAS and $B R A F$ mutations were found in 26.0 and $2.8 \%$ of CRCs, respectively. Between the two different PS groups, the distributions of $\mathrm{N}$ stage $(P=0.032)$, microsatellite instability (MSI) status $(P=0.035)$, and the ratio of tumor to normal lymph node LINE-1 methylation level (TNR) $(P=0.002)$ were different, but the other parameters were not (Table 1 ).

\section{Association between LINE-1 methylation and clinicopathological parameters}

To determine clinicopathological implications of the LINE-1 methylation status, we analyzed LINE-1 methylation in cancer and normal lymph node (LN) tissue samples. LINE-1 methylation levels in cancer tissue samples ranged from 29.81 to $78.73 \%$ (median, $52.64 \%$; standard deviation, $8.588 \%$ ), which was significantly lower than 
Table 1 Baseline characteristics of the epidemiological and clinical variables of the study population

\begin{tabular}{|c|c|c|c|c|c|c|}
\hline \multirow[t]{2}{*}{ Parameters } & \multicolumn{6}{|c|}{ ECOG performance status } \\
\hline & CaseN (\%) & ON (\%) & $\geq 1 N(\%)$ & $P$ value $^{a}$ & $P$ value $^{b}$ & OR \\
\hline \multicolumn{7}{|l|}{ Gender } \\
\hline Female & $125(37.2)$ & $64(38.6)$ & $61(35.9)$ & & & \\
\hline Male & $211(62.8)$ & $102(61.4)$ & $109(64.1)$ & 0.612 & 0.699 & 1.10 \\
\hline \multicolumn{7}{|l|}{ Age (median) } \\
\hline$<60$ years & $165(49.1)$ & $90(54.2)$ & $75(44.1)$ & & & \\
\hline$\geq 60$ years & $171(50.9)$ & $76(45.8)$ & $95(55.9)$ & 0.064 & 0.126 & 1.37 \\
\hline \multicolumn{7}{|l|}{ BMI } \\
\hline Normal & $135(40.2)$ & $74(44.6)$ & $61(35.9)$ & & & \\
\hline Overweight & $201(59.8)$ & $92(55.4)$ & $109(64.1)$ & 0.104 & 0.234 & 1.33 \\
\hline \multicolumn{7}{|l|}{ Tumor location } \\
\hline Proximal & $105(31.3)$ & $54(32.5)$ & $51(30.0)$ & & & \\
\hline Distal & $231(68.7)$ & $112(67.5)$ & $119(70.0)$ & 0.617 & 0.885 & 1.11 \\
\hline \multicolumn{7}{|l|}{ Pathology } \\
\hline Non-mucinous carcinoma & $321(95.5)$ & $160(96.4)$ & $161(94.7)$ & & & \\
\hline Mucinous adenocarcinoma & $15(4.5)$ & $6(3.6)$ & $9(5.3)$ & $0.599^{c}$ & 0.965 & 0.82 \\
\hline \multicolumn{7}{|l|}{ Differentiation } \\
\hline Low grade & $311(92.6)$ & $156(94.0)$ & 155 (91.2) & & & \\
\hline High grade & $25(7.4)$ & $10(6.0)$ & $15(8.8)$ & 0.328 & 0.185 & 2.51 \\
\hline \multicolumn{7}{|l|}{ T stage } \\
\hline $\mathrm{T} 1,2,3$ & $293(87.2)$ & 147 (88.6) & $146(85.9)$ & 0.464 & 0.568 & 1.08 \\
\hline $\mathrm{T} 4$ & $43(12.8)$ & 19 (11.4) & $24(14.1)$ & & & \\
\hline \multicolumn{7}{|l|}{ N stage } \\
\hline N 0,1 & $252(75.0)$ & $133(80.2)$ & $119(70.0)$ & 0.032 & 0.088 & 1.67 \\
\hline N 2 & $84(25.0)$ & $33(19.9)$ & $51(30.0)$ & & & \\
\hline \multicolumn{7}{|l|}{ Microsatellite status } \\
\hline MSS + MSI-L & $313(93.1)$ & $149(89.8)$ & $164(96.5)$ & 0.035 & 0.024 & 0.30 \\
\hline MSI-H & $21(6.3)$ & $15(9.0)$ & $6(3.5)$ & & & \\
\hline Missing & $2(0.6)$ & $2(1.2)$ & 0 & & & \\
\hline \multicolumn{7}{|l|}{ KRAS } \\
\hline Wild type & $230(68.5)$ & $122(73.5)$ & $108(63.5)$ & 0.342 & 0.367 & 1.38 \\
\hline Mutant type & $81(24.1)$ & $38(22.9)$ & $43(25.3)$ & & & \\
\hline Missing & $25(7.4)$ & $6(3.6)$ & $19(11.2)$ & & & \\
\hline \multicolumn{7}{|l|}{ BRAF } \\
\hline Wild type & $316(94.0)$ & $159(95.8)$ & 157 (92.4 \%) & 0.333 & 0.502 & 0.56 \\
\hline Mutant type & $9(2.7)$ & $6(3.6)$ & $3(1.8)$ & & & \\
\hline Missing & $11(3.3)$ & $1(0.6)$ & $10(5.9)$ & & & \\
\hline \multicolumn{7}{|l|}{ TNR (median) } \\
\hline$<0.69$ & $173(51.5)$ & $71(42.8)$ & $102(60.0)$ & 0.002 & 0.001 & 0.04 \\
\hline$\geq 0.69$ & $163(48.5)$ & $95(57.2)$ & $68(40.0)$ & & & \\
\hline
\end{tabular}

BMI body mass index, ECOG Eastern Cooperative Oncology Group, MSS microsatellite stable, MSI-L microsatellite instability-low, MSI- $H$ microsatellite instabilityhigh, TNR tumor to normal ratio

${ }^{a} \mathrm{Chi}$-square test

${ }^{\mathrm{b}}$ Unconditional logistic regression, adjusted for other selected covariates

'Fisher's exact test 
those of normal LN tissue samples which ranged from 48.50 to $89.90 \%$ (median, $76.80 \%$; standard deviation, $4.138 \%)(P<0.001)$. The TNR ranged from 0.63 to 0.78 with median of 0.69 (standard deviation, 0.117).

Association between LINE-1 methylation levels in cancer tissue samples and the PS of the patients were observed $(\mathrm{OR}=2.56, P<0.001)$. LINE-1 methylation levels in normal LNs were also associated with body mass index (BMI) status of the patient $(\mathrm{OR}=1.76, P=0.015)$. No association was found between other selected parameters, such as MSI status, and LINE-1 methylation levels of cancer or normal LN tissue samples (Table 2).

$L I N E-1$ methylation levels were significantly lower in cancer tissue samples of patients with $P S \geq 1$ than in those of patients with PS 0 (Fig. 1a). However, such a difference was not found in normal LN samples (Fig. 1b). The differences in LINE-1 methylation in cancer tissues between PS 0 and $P S \geq 1$ were significant in patients aged $\geq 60$ years, overweight patients (BMI $\geq 23.5$ ), and stage III cancers but not in patients aged $<60$ years, low BMI patients (BMI <23.5), and stage II cancers (Fig. 1c-e).
PS status, LINE-1 methylation status, and RFS

The median follow-up of the entire cohort at the time of data cut-off for the present analysis was 50.30 months, interquartile range (IQR) 46.68-53.92 months. At the last follow-up, a total of 41 disease-recurrence events (35 distant metastases and 6 local recurrences) were observed. The overall 4.5-year and 9-year recurrencefree survival (RFS) estimates were 87.50 and $83.00 \%$, respectively.

LINE-1 methylation was associated with RFS. The cutoff value for LINE-1 methylation levels was determined as $54.62 \%$ which was the median of LINE-1 methylation levels in the PS 0 group. This median value was close to the value $(54.12 \%)$ which generated the maximum value of Youden index used in conjunction with the receiver operating characteristic curve analysis. Low LINE-1 methylation status $(<54.62 \%)$ was closely associated with a shorter RFS time. The 4.5-year RFS was $93.9 \%$ in the high LINE-1 methylation patients, and it was $84.9 \%$ in the low LINE-1 methylation patients (log-rank test, $P=0.049$ ) (Fig. 2a). There was no significant difference in RFS

Table 2 The association between LINE-1 methylation status and clinical variables of the study population (stage III and high-risk stage $\|$ CRCs)

\begin{tabular}{|c|c|c|c|c|c|c|}
\hline \multirow[t]{3}{*}{ Parameters } & \multicolumn{6}{|c|}{ LINE-1 methylation levels (low vs. high) $^{a}$} \\
\hline & \multicolumn{3}{|c|}{ Cancer sample $(n=336)$} & \multicolumn{3}{|c|}{ Normal lymph node sample $(n=336)$} \\
\hline & $\mathrm{OR}$ & (95 \% C.I.) & $p^{\mathrm{b}}$ & $\mathrm{OR}$ & (95 \% C.I.) & $p^{b}$ \\
\hline \multicolumn{7}{|l|}{ Gender } \\
\hline (Male vs. female) & 1.49 & $(0.921-2.406)$ & 0.104 & 1.06 & $(0.670-1.677)$ & 0.803 \\
\hline \multicolumn{7}{|l|}{ Age } \\
\hline ( $\geq 60$ years vs. $<60$ years) & 1.20 & $(0.752-1.899)$ & 0.450 & 0.95 & $(0.611-1.480)$ & 0.823 \\
\hline \multicolumn{7}{|l|}{ BMI } \\
\hline (Overweight vs. normal) & 1.24 & $(0.775-1.996)$ & 0.366 & 1.76 & $(1.118-2.765)$ & 0.015 \\
\hline \multicolumn{7}{|l|}{ Tumor location } \\
\hline (Distal vs. proximal) & 0.81 & $(0.474-1.373)$ & 0.429 & 1.26 & $(0.762-2.079)$ & 0.370 \\
\hline \multicolumn{7}{|l|}{ Pathology } \\
\hline (Mucinous vs. non-mucinous) & 0.83 & $(0.196-3.521)$ & 0.802 & 1.44 & $(0.349-5.926)$ & 0.616 \\
\hline \multicolumn{7}{|l|}{ Differentiation } \\
\hline (High grade vs. low grade) & 0.37 & $(0.111-1.211)$ & 0.100 & 0.68 & $(0.213-2.173)$ & 0.515 \\
\hline \multicolumn{7}{|l|}{ T stage } \\
\hline (T4 vs. T1, 2, 3) & 0.81 & $(0.403-1.629)$ & 0.555 & 0.68 & $(0.347-1.324)$ & 0.255 \\
\hline \multicolumn{7}{|l|}{ N stage } \\
\hline (N2 vs. N 0,1$)$ & 1.24 & $(0.715-2.156)$ & 0.443 & 1.15 & $(0.681-1.938)$ & 0.602 \\
\hline \multicolumn{7}{|l|}{ Microsatellite status } \\
\hline (MSI-H vs. MSS + MSI-L) & 0.33 & $(0.106-1.008)$ & 0.052 & 0.76 & $(0.283-2.026)$ & 0.580 \\
\hline \multicolumn{7}{|l|}{ Performance status } \\
\hline$(P S \geq 1$ vs. $P S=0)$ & 2.56 & $(1.601-4.102)$ & $<0.001$ & 1.12 & $(0.718-1.752)$ & 0.615 \\
\hline
\end{tabular}

$B M I$ body mass index, MSI-H microsatellite instability, MSS microsatellite stable, MSI-L microsatellite instability-low, $P S$ performance status ${ }^{a}$ Cut-off points of LINE-1 methylation proportion were $54.62 \%$ in cancer tissue samples and $77.00 \%$ in normal lymph node samples

bunconditional logistic regression, adjusted for other selected covariates 

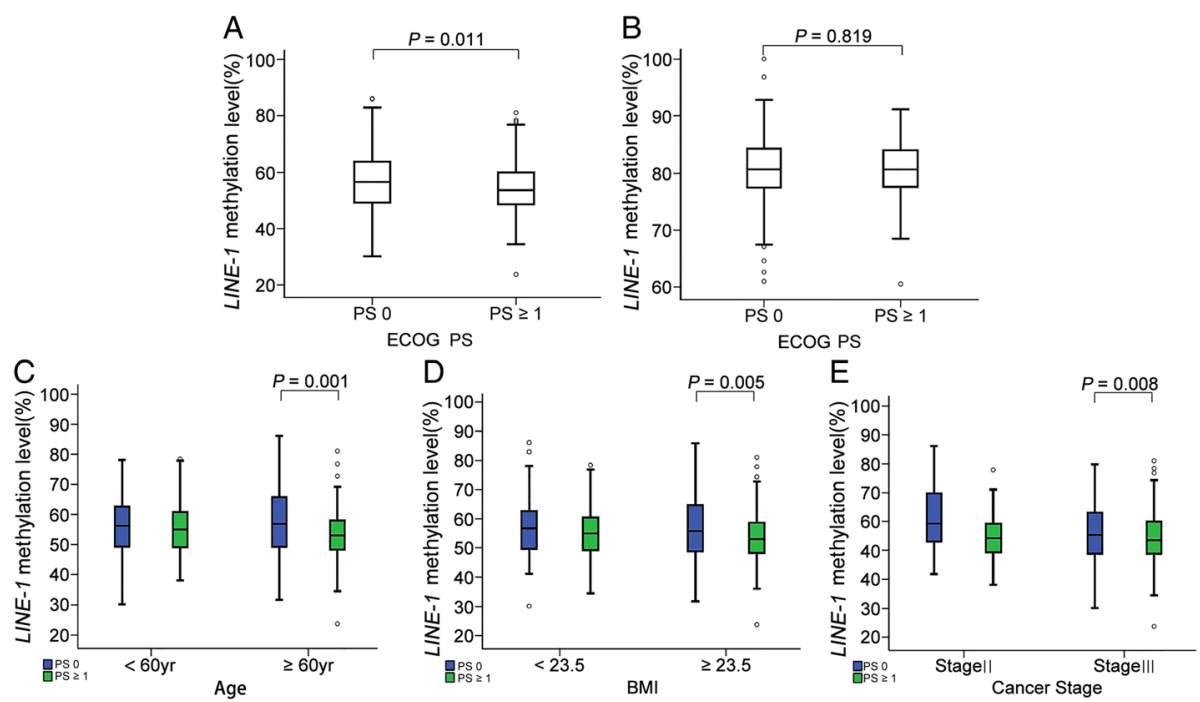

Fig. 1 Comparison of LINE-1 methylation levels between PS $=0$ and PS $\geq 1$ groups. $\mathbf{a}$, $\mathbf{b}$ LINE-1 methylation levels are significantly different between PS $=0$ and PS $\geq 1$ groups in cancer tissues (a) but not in normal lymph node samples (b). c-e Differences in LINE-1 methylation levels in cancer tissues between two PS groups were significant in patients who were older than 60 years (c), had a BMI indicating overweight (d), or stage III disease $(\mathbf{e})$
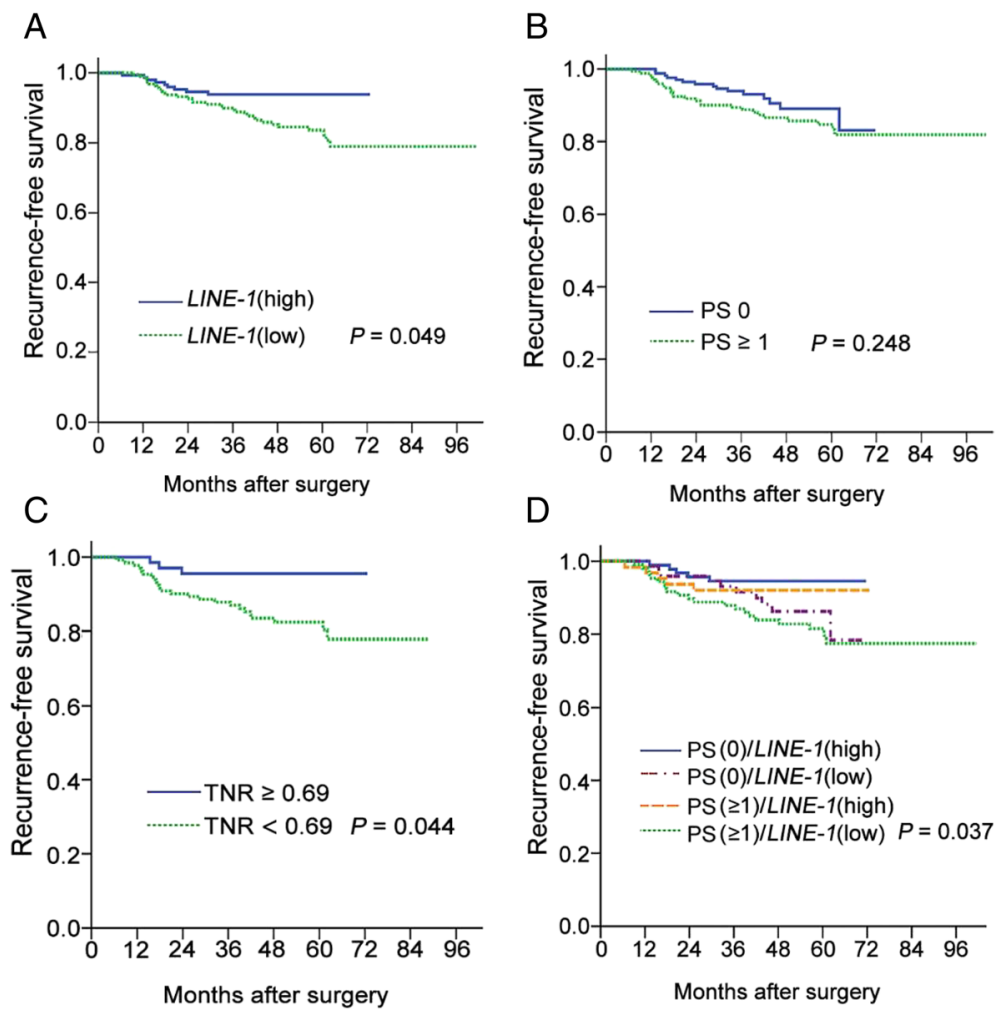

Fig. 2 Survival analyses. a Low LINE-1 methylation status was closely associated with shorter RFS times $(P=0.049)$. b There was no significant difference in recurrence-free survival according to the performance status (PS) score. c Survival analysis in patients with overweight BMI score according to the tumor to normal ratio (TNR) of LINE-1 methylation level. $\mathbf{d}$ In survival analysis stratified by combinatory PS and LINE-1 methylation statuses, recurrence-free survival was significantly different between PS(0)/LINE-1(high) and PS(1)/LINE-1(low) $(P=0.037)$ 
according to the PS: 4.5 -year RFS $93.9 \%$ in the PS 0 group and $85.7 \%$ in the PS $\geq 1 \quad(P=0.248$, Fig. $2 b)$. However, multivariate Cox regression analysis revealed that LINE-1 methylation status was not an independent prognostic parameter (Table 3).

We also performed survival analysis using the TNR value stratified by selected parameters such as age, gender, BMI, tumor location, $\mathrm{T}$ stage, $\mathrm{N}$ stage, and ECOG status. Low TNR value $(<0.69)$ was closely associated with a shorter RFS time in the overweight patients (higher BMI score) (Fig. 2c). The 4.5-year RFS was $95.6 \%$ in the high TNR value patients, and it was $83.8 \%$ in the low TNR value patients (log-rank test, $P=0.044$ ). The TNR value was not associated with other selected parameters.

When we counted RFS from the date of chemotherapy to the date of recurrence, Kaplan-Meier log-rank analysis showed survival curves similar to those using RFS calculated from the date of surgery to the date of recurrence by the Kaplan-Meier method with log-rank test (Additional file 1: Figure S1).

\section{Combinatory PS and LINE-1 methylation statuses as a predictor of recurrence in the setting of adjuvant FOLFOX} When we performed survival analysis with combinatory PS and LINE-1 methylation statuses, we found that the 4.5-year RFS was $94.5 \%$ in the PS(0)/LINE-1(high) group ( $n=93$ patients), $91.5 \%$ in the PS(0)/LINE-1(low) group ( $n=73$ ), $92.1 \%$ in the PS $(\geq 1) /$ LINE-1(high) group $(n=63)$, and $81.5 \%$ in the PS $(\geq 1) / L I N E-1$ (low) group $(n=107)$. The difference was significant between $\mathrm{PS}(0) / L I N E-1$ (high) and PS( $\geq 1$ )/LINE-1(low) (pairwise pooled log-rank test, $P=0.037$ ) (Fig. $2 \mathrm{~d}$ ). We did not perform further analyses of RFS in combination with
$B R A F$ and KRAS mutation statuses because of the low number of patients with this mutation.

Univariate analyses revealed that higher levels of $\mathrm{T}$ stage $(\mathrm{HR}=2.41,95 \%$ C.I. $=1.179-4.908, P=0.016), \mathrm{N}$ stage $(\mathrm{HR}=2.51,95 \%$ C.I. $=1.355-4.654, P=0.003)$ and $\mathrm{PS}(\geq 1) /$ LINE-1 (low) $(\mathrm{HR}=2.72,95 \%$ C.I. $=1.013-7.325$, $P=0.047)$ were associated with higher recurrence. Multivariable Cox regression analyses adjusting for gender, age, BMI, tumor location, histology, $\mathrm{T}$ stage, $\mathrm{N}$ stage, and microsatellite instability status revealed consistent statistical patterns and $P$ values in $\mathrm{T}$ stage $(\mathrm{HR}=2.60$, $95 \%$ C.I. $=1.251-5.413, P=0.011)$ and $\mathrm{N}$ stage $(\mathrm{HR}=$ $2.78,95 \%$ C.I. $=1.429-5.393, P=0.003)$, but $\mathrm{PS}(\geq 1) /$ LINE-1(low) status was not associated with RFS (HR = 2.27, $95 \%$ C.I. $=0.839-6.168, P=0.106)$ (Table 4$)$.

\section{Prognostic value of LINE-1 methylation status alone or combined PS and LINE-1 statuses in stage III colon cancers}

Because high-risk stage II CRCs or stage III rectal cancers may differ from stage III colon cancers with respect to the beneficial effect from adjuvant FOLFOX vs. 5-FU/ leucovorin, survival analysis was needed to be performed in stage III colon cancers with exclusion of rectal cancers and stage II colon cancers. The associations between LINE-1 methylation levels with covariates investigated in the study population (stage III colon cancers only) (Table 5) were similar to those between LINE1 methylation levels with covariates investigated in the study population (stage III colon cancers plus high-risk stage II CRCs and stage III rectal cancers) (Table 2). Multivariate Cox regression analysis revealed that low LINE-1 methylation status was associated with a shorter RFS time (Table 6). PS( $\geq 1$ )/LINE-1(low) was

Table 3 Impact of clinicopathological parameters, including performance status and LINE-1 methylation status, on cancer recurrence in stage III or high-risk stage II CRCS $(n=336)$, assessed by univariate and multivariate Cox regression analyses

\begin{tabular}{|c|c|c|c|c|c|c|}
\hline \multirow[t]{2}{*}{ Parameters } & \multicolumn{3}{|c|}{ Univariate analysis } & \multicolumn{3}{|c|}{ Multivariate analysis } \\
\hline & $\mathrm{HR}$ & $95 \%$ C.I. & $P^{a}$ & $\mathrm{HR}$ & $95 \%$ C.I. & $P^{a}$ \\
\hline Gender (male vs. female) & 1.66 & $0.834-3.321$ & 0.149 & & & \\
\hline Age ( $\geq 60$ years vs. $<60$ years) & 1.45 & $0.774-2.719$ & 0.245 & & & \\
\hline BMI (overweight vs. normal) & 1.29 & $0.674-2.454$ & 0.445 & & & \\
\hline Tumor location (distal vs. proximal) & 1.94 & $0.897-4.205$ & 0.092 & & & \\
\hline Pathology (mucinous vs. non-mucinous) & 1.29 & $0.311-5.343$ & 0.726 & & & \\
\hline Differentiation (high grade vs. low grade) & 1.15 & $0.353-3.718$ & 0.821 & & & \\
\hline T stage (T4 vs. T1, 2, 3) & 2.41 & $1.179-4.908$ & 0.016 & 2.33 & $1.142-4.761$ & 0.020 \\
\hline N stage (N 2 vs. N0, 1) & 2.51 & $1.355-4.654$ & 0.003 & 2.46 & $1.328-4.569$ & 0.004 \\
\hline Microsatellite status (MSI-H vs. MSS + MSI-L) & 1.24 & $0.381-4.002$ & 0.725 & & & \\
\hline Performance status (PS $\geq 1$ vs. PS =0) & 1.46 & $0.766-2.770$ & 0.251 & & & \\
\hline LINE-1 methylation (low vs. high) & 2.15 & $0.984-4.701$ & 0.055 & & & \\
\hline
\end{tabular}


Table 4 Univariate and multivariate Cox regression analyses for recurrence-free survival in stage III or high-risk stage II CRCs $(n=336)$

\begin{tabular}{|c|c|c|c|c|c|c|}
\hline \multirow[t]{2}{*}{ Parameters } & \multicolumn{3}{|c|}{ Univariate analysis } & \multicolumn{3}{|c|}{ Multivariate analysis } \\
\hline & $\overline{H R}$ & $95 \%$ C.I. & $P$ & $\overline{\mathrm{HR}}$ & $95 \%$ C.I. & $p^{a}$ \\
\hline T stage (T4 vs. T1, 2, 3) & 2.41 & $1.179-4.908$ & 0.016 & 2.36 & $1.153-4.818$ & 0.019 \\
\hline N stage (N 2 vs. N0, 1) & 2.51 & $1.355-4.654$ & 0.003 & 2.43 & $1.309-4.513$ & 0.005 \\
\hline \multicolumn{7}{|c|}{ Performance status_LINE-1 methylation } \\
\hline PS(0)/LINE-1 (high) & Ref. & & & Ref. & & \\
\hline $\mathrm{PS}(0) / L I N E-1$ (low) & 2.02 & $0.684-5.936$ & 0.203 & 2.07 & $0.704-6.105$ & 0.185 \\
\hline $\operatorname{PS}(\geq 1) / L I N E-1$ (high) & 1.25 & $0.360-4.337$ & 0.343 & 1.20 & $0.344-4.152$ & 0.778 \\
\hline PS $(\geq 1) / L I N E-1$ (low) & 2.72 & $1.013-7.325$ & 0.047 & 2.59 & $0.966-6.964$ & 0.059 \\
\hline
\end{tabular}

BMI body mass index, ECOG Eastern Cooperative Oncology Group, MSS microsatellite stable, MSI-L microsatellite instability-low, MSI-H microsatellite instabilityhigh, PS performance status

${ }^{a}$ Cox proportional hazard regression model, adjusted for other selected covariates

independently associated with disease recurrence (HR $=5.06$, $95 \%$ C.I. $=1.142-22.377, P=0.033)$ in multivariate analysis (Table 7 ).

\section{Discussion}

The present study has demonstrated that ECOG-PS was associated with $L I N E-1$ methylation in cancer tissue. The difference in $L I N E-1$ methylation in cancer tissue between PS 0 and PS $\geq 1$ was significant in patients of older age, higher BMI score, or stage III disease. LINE-1 methylation levels change with aging and disease progression, and our stratified analyses of $L I N E-1$ methylation levels adjusting for potential confounding suggested that low levels of LINE-1 methylation had a closer relationship with poorer PS. To our knowledge, the present study is the first study to assess the association between PS statuses of CRC patients and LINE-1 methylation levels of cancer tissue.
A previous study suggests that LINE-1 methylation is a strong, independent predictor of recurrence-free survival in adjuvant FOLFOX-treated stage III colon cancers [32]. Our present study also showed that low LINE-1 methylation status was closely associated with shorter RFS time. However, PS was not associated with RFS. This result was not consistent with previous studies showing that PS was predictive of survival in patients with locally advanced or metastatic carcinoma [38, 39]. There are several factors triggering this result. First, this result may be due to the small number of relapse events and the follow-up of RFS. The Kaplan-Meier analysis showed that the curves of PS 0 and PS $\geq 1$ separated early, and most events happened before the median follow-up time, which is an inaccurate measurement of the median RFS. Another reason may be the limited PS subgroups in the study. PS was distributed into mainly

Table 5 The association between LINE-1 methylation status and clinicopathological parameters (stage III colon cancers only)

\begin{tabular}{|c|c|c|c|c|c|c|}
\hline \multirow[t]{3}{*}{ Parameters } & \multicolumn{6}{|c|}{ LINE-1 methylation levels (low vs.high) } \\
\hline & \multicolumn{3}{|c|}{ Cancer sample $(n=268)$} & \multicolumn{3}{|c|}{ Normal LN sample $(n=268)$} \\
\hline & $\overline{O R}$ & (95 \% C.I.) & $P^{a}$ & $\overline{O R}$ & $(95 \%$ C.I. $)$ & $P^{a}$ \\
\hline Gender (male vs. female) & 1.63 & $(0.971-2.747)$ & 0.065 & 1.11 & $(0.699-1.745)$ & 0.670 \\
\hline Age ( $\geq 60$ years vs. $<60$ years) & 1.23 & $(0.742-2.053)$ & 0.419 & 0.97 & $(0.624-1.508)$ & 0.893 \\
\hline BMI (overweight vs. normal) & 1.21 & $(0.711-2.044)$ & 0.487 & 1.56 & $(0.991-2.444)$ & 0.055 \\
\hline Tumor location (distal vs. proximal) & 0.78 & $(0.438-1.383)$ & 0.393 & 1.14 & $(0.694-1.887)$ & 0.598 \\
\hline \multicolumn{7}{|l|}{ Pathology } \\
\hline (Mucinous vs. non-mucinous) & 0.77 & $(0.149-4.004)$ & 0.759 & 1.52 & $(0.361-6.387)$ & 0.569 \\
\hline \multicolumn{7}{|l|}{ Differentiation } \\
\hline (High grade vs. low grade) & 0.58 & $(0.154-2.185)$ & 0.420 & 0.51 & $(0.154-1.687)$ & 0.270 \\
\hline T stage (T4 vs. T1, 2, 3) & 0.99 & $(0.417-2.359)$ & 0.985 & 0.74 & $(0.381-1.447)$ & 0.382 \\
\hline N stage (N2 vs. N0, 1) & 1.07 & $(0.600-1.921)$ & 0.812 & 1.21 & $(0.720-2.039)$ & 0.470 \\
\hline \multicolumn{7}{|l|}{ Microsatellite status } \\
\hline (MSI-H vs. MSS + MSI-L) & 0.40 & $(0.118-1.388)$ & 0.150 & 1.02 & $(0.380-2.728)$ & 0.972 \\
\hline Performance status (PS $\geq 1$ vs. $P S=0$ ) & 1.80 & $(1.081-3.004)$ & 0.024 & 1.20 & $(0.770-1.871)$ & 0.421 \\
\hline
\end{tabular}

$B M I$ body mass index, MSS microsatellite stable, MSI- $L$ microsatellite instability-low, MSI-H microsatellite instability-high, $P S$ performance status

anconditional logistic regression, adjusted for other selected covariates 
Table 6 Univariate and multivariate Cox regression analyses for recurrence-free survival in stage III colon cancers $(n=268)$

\begin{tabular}{|c|c|c|c|c|c|c|}
\hline \multirow[t]{2}{*}{ Parameters } & \multicolumn{3}{|c|}{ Univariate analysis } & \multicolumn{3}{|c|}{ Multivariate analysis } \\
\hline & $\overline{\mathrm{HR}}$ & $95 \%$ C.I. & $P^{a}$ & $\overline{\mathrm{HR}}$ & $95 \%$ C.I. & $P^{a}$ \\
\hline Gender (male vs. female) & 1.96 & $0.881-4.367$ & 0.099 & & & \\
\hline Age ( $\geq 60$ years vs. $<60$ years) & 1.54 & $0.775-3.161$ & 0.234 & & & \\
\hline BMI (overweight vs. normal) & 2.23 & $0.966-5.164$ & 0.060 & & & \\
\hline Tumor location (distal vs. proximal) & 2.32 & $0.955-5.637$ & 0.063 & & & \\
\hline Pathology (mucinous vs. non-mucinous) & 1.71 & $0.409-7.166$ & 0.462 & & & \\
\hline Differentiation (high grade vs. low grade) & 1.41 & $0.428-4.619$ & 0.575 & & & \\
\hline T stage (T4 vs. T1, 2, 3) & 2.97 & $1.284-6.865$ & 0.011 & 2.50 & $1.065-5.854$ & 0.035 \\
\hline N stage (N2 vs. N0, 1) & 2.40 & $1.196-4.795$ & 0.014 & 2.26 & $1.114-4.575$ & 0.024 \\
\hline Microsatellite status (MSI-H vs. MSS + MSI-L) & 1.06 & $0.254-4.451$ & 0.933 & & & \\
\hline Performance status (PS $\geq 1$ vs. PS 0) & 1.63 & $0.782-3.394$ & 0.192 & & & \\
\hline LINE-1 methylation (low vs. high) & 3.36 & $1.287-8.781$ & 0.013 & 3.50 & $1.338-9.126$ & 0.011 \\
\hline
\end{tabular}

$B M I$ body mass index, MSS microsatellite stable, MSI-L microsatellite instability-low, MSI-H microsatellite instability-high, $P S$ performance status

${ }^{a}$ Cox proportional hazard regression model, adjusted for other selected covariates

two groups: PS 0 and PS 1 , with only one patient having PS 2. The lack of patients with PS $>1$ limited the power to distinguish significant differences in RFS with different PS groups. Because the result was not significant in our condition, we performed statistical power analyses to decide the likelihood that the test can detect effects of a given size in this particular situation. The power analysis found that the overall 336 subjects (170 in PS $\geq 1$ and 166 in PS 0) achieved a $56.93 \%$ power at a 0.050 significance level to detect a difference in RFS between $\mathrm{PS} \geq 1$ and PS 0 (Additional file 2: Figure S2A). The sample size estimation suggested that a larger number should be included in further study if the relapse events are rare while the relapse-free proportion stays high during follow-up of the PS $\geq 1$ group. The stronger power of the precision to provide reliable answers requires larger sample numbers (Additional file 2: Figure S2B).

Our results suggested that RFS was highest in CRCs with PS(0)/LINE-1(high) and lowest in CRCs with PS( $\geq 1$ )/LINE-1(low). Therefore, we just found a significant difference between the two groups of CRCs in univariate analysis. This difference was not significant in multivariate analyses. This lack of significance may be affected by other parameters that are involved in multivariate analysis and the small number of patients in each PS/LINE-1 groups after stratification. However, in stage III colon cancer patients with exclusion of rectal cancers and high-risk stage II colon cancers patients, not only LINE-1 methylation status alone but also PS $(\geq 1) / L I N E$-1(low) was independently associated with disease recurrence in multivariate analysis. Whether PS( $\geq 1$ )/LINE-1(low) might identify a subset of patients with poor prognosis is required to be validated with a large-size samples of stage III colon cancers.

The TNR value is more appealing parameter compared with just methylation level of LINE-1 measured in cancer tissue samples. The value reflects methylation change in tumor compared with matched normal tissue and removes some artifacts inherent using FFPE samples and methylation analysis technology. It provides possible normalization of many confounding variables in the analysis technology. We found that low TNR value was

Table 7 Univariate and multivariate Cox regression analyses for recurrence-free survival in stage III colon cancers only $(n=268)$

\begin{tabular}{|c|c|c|c|c|c|c|}
\hline \multirow[t]{2}{*}{ Parameters } & \multicolumn{3}{|c|}{ Univariate analysis } & \multicolumn{3}{|c|}{ Multivariate analysis } \\
\hline & $\mathrm{HR}$ & $95 \%$ C.I. & $p^{a}$ & $H R$ & $95 \%$ C.I. & $p^{a}$ \\
\hline T stage (T1, 2, 3 vs. T4) & 2.97 & $1.284-6.865$ & 0.011 & 2.97 & $1.284-6.865$ & 0.036 \\
\hline $\mathrm{N}$ stage (N0, 1 vs. N2) & 2.40 & $1.196-4.795$ & 0.014 & 2.40 & $1.196-4.795$ & 0.023 \\
\hline \multicolumn{7}{|c|}{ Performance status — LINE-1 methylation } \\
\hline PS(0)/LINE-1 (high) & Ref. & & & Ref. & & \\
\hline$P S(0) / L I N E-1$ (low) & 3.69 & $0.794-17.167$ & 0.096 & 3.69 & $0.794-17.167$ & 0.082 \\
\hline $\operatorname{PS}(\geq 1) / L I N E-1$ (high) & 1.78 & $0.297-10.678$ & 0.528 & 1.78 & $0.297-10.678$ & 0.528 \\
\hline$P S(\geq 1) /$ LINE-1 (low) & 5.20 & $1.197-22.611$ & 0.028 & 5.20 & $1.197-22.611$ & 0.025 \\
\hline
\end{tabular}

PS performance status

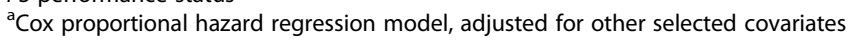


closely associated with a shorter RFS time in the overweight patient.

Measurement of LINE-1 methylation level in bisulfitetreated genomic DNA samples using MethyLight or pyrosequencing assay has been shown to correlate with genomic DNA methylation levels measured by liquid chromatography-mass spectrometry [40, 41], which provided a basis for use of LINE-1 pyrosequencing methylation assay as a surrogate marker for genomic DNA methylation level. The precision and reliability of LINE-1 pyrosequencing assay have been shown in Irahara et al.'s study which evaluated performance of pyrosequencing assay of LINE-1 methylation levels in genomic DNA samples from formalin-fixed paraffin-embedded (FFPE) tissue samples, fresh-frozen tissue samples, and peripheral blood leukocytes [42]. Our study measured the same four CpG sites which Irahara et al.'s study assayed using pyrosequencing. However, Tournier et al. raised a doubt upon results of pyrosequencing methylation assay in FFPE tissue samples because they found discrepant methylation levels in paired fresh-frozen and FFPE tissue samples [43]. In our previous study [44], we analyzed paired fresh-frozen and FFPE tissue samples for their methylation level at the four CPG sites of LINE-1 and found that FFPE tissue samples tended to show increased methylation levels at four CpG sites compared with fresh tissue samples although methylation levels of individual CpG sites showed strong correlations between paired fresh-frozen and FFPE tissue samples. The foldincrease was similar at CpG sites 2 and 3 but different from that of CpG site 1 or 4 [44], and thus, we determined to obtain average value of methylation levels at CpG sites 2 and 3 as representative value of LINE-1 methylation levels in FFPE tissue samples.

The present study has several limitations. The major limitation was that, because all the patients were treated with adjuvant FOLFOX, we could not evaluate the interaction between LINE-1 methylation and treatment effect of adjuvant FOLFOX. Nonetheless, our cohort was relatively homogeneous in stage (stage III and high-risk stages II included) and treatment (surgery and adjuvant FOLFOX at a single institution). Another limitation is the relatively short duration of follow-up. However, the median follow-up duration exceeded 4.5 years, and RFS at which point exhibits a good correlation with longer follow-up periods of overall survival (OS) in colon cancer. Further analyses, including determinations of the OS, will be performed in the future after longer durations of follow-up.

\section{Conclusions}

We found that PS was associated with LINE-1 methylation in CRC tissue. For stage III colon cancer patients treated with adjuvant FOLFOX, LINE-1 methylation was associated with RFS. Patients with tumors having concurrent PS $(\geq 1) / L I N E-1$ (low) had a higher recurrence rate. Further validation and translational studies to improve treatment outcomes in this subset of patients will be needed in the future. However, LINE-1 methylation status and combined PS and LINE-1 statuses were not prognostic parameters in stage III or high-risk stage II CRCs treated with adjuvant FOLFOX.

\section{Methods \\ Patients}

A total of 336 CRC patients who received curative surgery and adjuvant chemotherapy in the Seoul National University Hospital, Seoul, Korea, from June 2005 to November 2011 were included. The following eligibility criteria were used for this retrospective study: age at diagnosis $>18$ years, stage III (any T and N1 or N2M0) or high-risk stage II (T3 or T4N0M0), completion of at least 6 cycles of adjuvant FOLFOX, and adequate organ functions. High risk was defined as follows: T4 tumor, poor histological grade, lymphovascular invasion, bowel obstruction at presentation, and localized perforation. Exclusion criteria were the following: previous chemotherapy for CRC, previous radiotherapy for CRC, signet ring cell histology, distant metastasis, and history of other malignancies within the previous 5 years. Hereditary non-polyposis colon cancer syndrome or familial adenomatous polyposis was excluded. Patients received either FOLFOX-4 (165 patients) or modified FOLFOX-6 (171 patients). Each cycle of FOLFOX-4 consisted of oxaliplatin $\left(85 \mathrm{mg} / \mathrm{m}^{2}\right)$ on day 1 and folinic acid $\left(200 \mathrm{mg} / \mathrm{m}^{2}\right)$ and a bolus of $5-\mathrm{FU}\left(400 \mathrm{mg} / \mathrm{m}^{2}\right)$ followed by a 22 -h infusion of 5 -FU $\left(600 \mathrm{mg} / \mathrm{m}^{2}\right)$ on days 1 and 2 , which was repeated every 2 weeks. Modified FOLFOX-6 consisted of oxaliplatin $\left(85 \mathrm{mg} / \mathrm{m}^{2}\right)$, folinic acid $\left(400 \mathrm{mg} / \mathrm{m}^{2}\right)$, and a bolus of 5 -FU $\left(400 \mathrm{mg} / \mathrm{m}^{2}\right)$ followed by a $46-\mathrm{h}$ infusion of 5 -FU $\left(2400 \mathrm{mg} / \mathrm{m}^{2}\right)$ repeated every 2 weeks. Adjuvant chemotherapy was continued until completion of the planned 12 cycles, recurrence, toxicity, or patient refusal. In 336 patients, $303(90.2 \%)$ patients had completed all 12 cycles of adjuvant chemotherapy. Thirty-three $(9.8 \%)$ patients completed more than 6 cycles but less than 12 cycles of adjuvant chemotherapy. Computed tomography (CT) imaging was performed every six cycles during the chemotherapy period, and patients were followed up at least every 6 months after completion of the chemotherapy. Recurrence was defined based on CT scans in the case of distant metastasis, and the treating oncologist made the decision to perform pathological confirmation when necessary.

The study protocol was reviewed and approved by the institutional review board of Seoul National University Hospital (1408-075-604). Informed consent was exempted 
because of the retrospective nature of the study and minimal risk of harm to the study subjects. This study was performed in accordance with the recommendations of the Declaration of Helsinki for biomedical research involving human subjects. Patient records/information was anonymized and de-identified prior to analysis.

\section{Molecular pathologic analyses}

Analyses of DNA methylation and microsatellite instability (MSI) were performed as described previously $[17,45]$. Briefly, tissue slides were reviewed, and the areas of high tumor cell density $\left(1 \mathrm{~cm}^{2}\right)$ were marked and dissected using a knife blade. Normal abdominal LN tissues from the cancer patients were also dissected. Dissected tissues were collected into a microtube containing tissue lysis buffer and proteinase K. The EZ DNA methylation kit was used to convert DNA using sodium bisulfite (Zymo Research, Orange, CA), and LINE-1 methylation levels were measured using a pyrosequencing methylation assay. We used the same oligonucleotide primers as the ones designed by the Issa group [46]. The primers and PCR conditions are listed in Additional file 3: Table S1. The LINE-1 assays were performed in a 25$\mu \mathrm{L}$ PCR reaction containing $2-\mu \mathrm{L}$ bisulfite-treated genomic DNA, $60 \mathrm{mM}$ Tris- $\mathrm{HCl}(\mathrm{pH} 8.8), 15 \mathrm{mM}$ ammonium sulfate, $0.5 \mathrm{mM} \mathrm{MgCl}_{2}, 1 \mathrm{mM}$ dNTP mix, and $1 \mathrm{U}$ of Taq polymerase. The PCR products were purified and quantified in the PyroMark Q24 System (Biotage $\mathrm{AB}$, Uppsala, Sweden). The amounts of $\mathrm{C}$ nucleotides relative to the sum of $C$ and $T$ nucleotides at each $\mathrm{CpG}$ site were calculated as percentages. The average of two percentage values in the two adjacent CpG sites (nucleotide positions 321 and 306 of X58075 (GenBank)) was taken as the overall LINE-1 methylation level in a given sample. The standard deviations for these calculations were given as a supplementary file. For cancer samples, the cut-off values for high and low methylation statuses were set at the median value of LINE-1 methylation levels in CRCs of PS 0 patients, whereas for lymph node samples, the cut-off values was set at the median value of LINE-1 methylation levels in LN samples of all the patients.

The MSI status of each tumor was determined using National Cancer Institute's five consensus microsatellite markers (D2S123, D5S346, D17S250, BAT25, and BAT26). Either the forward or reverse primer for each marker was labeled with fluorescence, and PCR products were electrophoresed and analyzed. We classified MSI status as follows: MSI-high (instability at $\geq 2$ microsatellite markers), MSI-low (instability at 1 marker), or MSS (instability at none).

$B R A F$ mutations at codon 600 (V600E) and KRAS mutations at codons 12 and 13 were analyzed by a real-time polymerase chain reaction-based allelic discrimination method and direct sequencing, respectively, as previously described [47].

\section{ECOG-PS measurement}

PS was measured at the start of chemotherapy using the ECOG-PS, which is an ordinal scale with scores from 0 to 5 [48]: 0 , normal activity; 1 , symptomatic but ambulatory; 2 , symptomatic - confined to bed/chair $<50 \%$ of waking hours; 3 , symptomatic-confined to bed/chair $>50 \%$ of waking hours; 4, $100 \%$ bedridden; and 5, dead. The present study included 166 cases with PS 0, 169 cases with PS 1 , and 1 case with PS 2 . For all CRC patients, the PS scores were rated before initiation of adjuvant therapy.

\section{Statistical analysis}

Post hoc exploratory analysis was performed using individual methylation markers. The number of methylation loci was compared using Student's $t$ test or one-way analysis of variance (ANOVA). Categorical variables were compared using chi-square test or Fisher's exact test. TNR of LINE-1 methylation level was calculated by the equation (= (average methylation level at LINE-1 CpG sites 2 and 3 in tumor)/ (average methylation level at LINE-1 CpG sites 2 and 3 in normal $\mathrm{LN}$ )). Unconditional logistic regression analysis was performed to measure the association between LINE-1 methylation and clinicopathological parameters. Clinical data were last updated in November 2014. RFS was calculated from the date of surgery to the date of recurrence or death by the Kaplan-Meier method with log-rank test. To adjust for baseline characteristics, we performed univariate and multivariate analysis using a Cox proportional hazard model. Age, gender, stages (II vs. III), histology (mucinous adenocarcinoma vs. others), tumor location (proximal vs. distal), and MSI status (MSI-high vs. others) were included as covariates. The reason why MSI was put into multivariate analysis include as follows: (1) that MSI is known to be one of the prognostic biomarkers, (2) that MSI status is associated with tumoral LINE-1 methylation levels [49], and (3) that survival of patients with MSI-high CRCs depends on tumoral LINE-1 methylation status [50]. Twosided $P$ values of $<0.05$ were considered significant in all analyses. Statistical analyses were performed using SPSS software for Windows, version 17.0 (SPSS, Chicago, IL, USA), but the sample size and power estimation of this study was calculated using PASS software (PASS 08) (NCSS, UT, USA). The Youden index in conjunction with the receiver operating characteristic curve analysis was estimated using SAS software (version 9.3 for Microsoft Windows; SAS Institute, Cary, NC, USA). 


\section{Availability of supporting data}

The datasets supporting the results of this article are included within the article.

\section{Additional files}

Additional file 1: Figure S1. Survival analyses using RFS from the date of chemotherapy. (A) Survival analyses show that low LINE-1 methylation status was closely associated with shorter RFS times $(P=0.040)$. (B) There was no significant difference in RFS according to the performance status (PS). (C) Survival analysis stratified by combinatory PS and LINE-

1 methyaltion statuses. The difference was significant between PS(0)/LINE1 (high) and PS(1)/LINE-1(low) $(P=0.021)$. (D) Survival analysis using the

TNR value in higher BMI score patients. (TIF $3476 \mathrm{~kb}$ )

Additional file 2: Figure S2. The power and sample size analysis. (A) Overall, 336 subjects (170 in PS $\geq 1$ and 166 in PS =0) achieved $56.93 \%$ power at a 0.050 significance level to detect differences RFS between $P S \geq 1$ and $P S=0$ groups. (B) The sample size soars as power of ECOG 1 relapse-free proportion increases. (TIF $948 \mathrm{~kb}$ )

Additional file 3: Table S1. Primer sequences and PCR conditions used for pyrosequencing. (DOC $27 \mathrm{~kb}$ )

\section{Competing interests}

The authors declare that they have no competing interests.

\section{Authors' contributions}

DC, XW, and GHK were involved in the study concept and design. DC, XW, YSS, YYR, THL, NYC, SWH, TYK, and GHK involved in the data acquisition. The analysis and interpretation of data were done by DC and XW. The manuscript writing was made by DC and GHK. The manuscript review and final approval were done by DC, XW, YSS, YYR, THL, NYC, SWH, TYK, and GHK. All authors read and approved the final manuscript.

\section{Acknowledgements}

This study was supported by a grant from the Basic Science Research Program through the National Research Foundation of Korea (NRF) funded by the Ministry of Education (2013R1A1A2059080); a grant from the Priority Research Centers Program through the National Research Foundation of Korea (NRF) funded by MEST (2009-0093820); the NRF grant funded by the Ministry of Science, ICT, and Future Planning (MSIP) (2011-0030049); and a grant from the Korean Health Technology R\&D Project through the Korea Health Industry Development Institute (KHIDI), funded by the Ministry of Health \& Welfare, Republic of Korea (HI14C1277).

\section{Author details}

${ }^{1}$ Key Laboratory of Carcinogenesis and Translational Research (Ministry of Education), Department of Cancer Epidemiology, Peking University Cancer Hospital and Institute, Beijing, China. ${ }^{2}$ Department of Pathology, Seoul National University College of Medicine, Seoul, Korea. ${ }^{3}$ Laboratory of Epigenetics, Cancer Research Institute, Seoul National University College of Medicine, Seoul, Korea. ${ }^{4}$ Division of Oncology, Department of Internal Medicine, Seoul National University College of Medicine, Seoul, Korea.

Received: 15 December 2015 Accepted: 31 March 2016

Published online: 05 April 2016

\section{References}

1. Jemal A, Bray F, Center MM, Ferlay J, Ward E, Forman D. Global cancer statistics. CA Cancer J Clin. 2011;61(2):69-90. doi:10.3322/caac.20107.

2. Center MM, Jemal A, Smith RA, Ward E. Worldwide variations in colorectal cancer. CA Cancer J Clin. 2009;59(6):366-78. doi:10.3322/caac.20038.

3. Park S, Shin HR, Lee B, Shin A, Jung KW, Lee DH, et al. Attributable fraction of alcohol consumption on cancer using population-based nationwide cancer incidence and mortality data in the Republic of Korea. BMC Cancer. 2014:14:420. doi:10.1186/1471-2407-14-420.

4. Andre T, Boni C, Mounedji-Boudiaf L, Navarro M, Tabernero J, Hickish T, et al. Oxaliplatin, fluorouracil, and leucovorin as adjuvant treatment for colon cancer. N Engl J Med. 2004;350(23):2343-51. doi:10.1056/NEJMoa032709.
5. Andre T, Boni C, Navarro M, Tabernero J, Hickish T, Topham C, et al. Improved overall survival with oxaliplatin, fluorouracil, and leucovorin as adjuvant treatment in stage II or III colon cancer in the MOSAIC trial. J Clin Oncol. 2009:27(19):3109-16. doi:10.1200/JCO.2008.20.6771.

6. Lengauer C, Kinzler KW, Vogelstein B. DNA methylation and genetic instability in colorectal cancer cells. Proc Natl Acad Sci U S A. 1997;94(6):2545-50.

7. Lander ES, Linton LM, Birren B, Nusbaum C, Zody MC, Baldwin J, et al. Initial sequencing and analysis of the human genome. Nature. 2001;409(6822):860-921. doi:10.1038/35057062.

8. Hormozdiari F, Alkan C, Ventura M, Hajirasouliha I, Malig M, Hach F, et al. Alu repeat discovery and characterization within human genomes. Genome Res. 2011;21(6):840-9. doi:10.1101/gr.115956.110.

9. Yoder JA, Walsh CP, Bestor TH. Cytosine methylation and the ecology of intragenomic parasites. Trends Genet. 1997:13(8):335-40.

10. Chalitchagorn K, Shuangshoti S, Hourpai N, Kongruttanachok N, Tangkijvanich $\mathrm{P}$, Thong-ngam D, et al. Distinctive pattern of LINE-1 methylation level in normal tissues and the association with carcinogenesis. Oncogene. 2004:23(54):8841-6. doi:10.1038/sj.onc.1208137.

11. Sunami $E$, de Maat M, Vu A, Turner RR, Hoon DS. LINE-1 hypomethylation during primary colon cancer progression. PLoS One. 2011;6(4):e18884. doi:10.1371/journal.pone.0018884.

12. van Hoesel AQ, van de Velde CJ, Kuppen PJ, Liefers GJ, Putter H, Sato Y, et al. Hypomethylation of LINE-1 in primary tumor has poor prognosis in young breast cancer patients: a retrospective cohort study. Breast Cancer Res Treat. 2012;134(3):1103-14. doi:10.1007/s10549-012-2038-0.

13. Saito K, Kawakami K, Matsumoto I, Oda M, Watanabe G, Minamoto T. Long interspersed nuclear element 1 hypomethylation is a marker of poor prognosis in stage IA non-small cell lung cancer. Clin Cancer Res. 2010;16(8):2418-26.

14. Li J, Huang Q, Zeng F, Li W, He Z, Chen W, et al. The prognostic value of global DNA hypomethylation in cancer: a meta-analysis. PLoS One. 2014;9(9):e106290. doi:10.1371/journal.pone.0106290.

15. Zelic R, Fiano V, Grasso C, Zugna D, Pettersson A, Gillio-Tos A, et al. Global DNA hypomethylation in prostate cancer development and progression: a systematic review. Prostate Cancer Prostatic Dis. 2015;18(1):1-12. doi:10.1038/pcan.2014.45.

16. Tang JT, Wang ZH, Fang JY. Assessing the potential value of long interspersed element-1 hypomethylation in colorectal cancer: evidence from retrospective studies. Onco Targets Ther. 2015:8:3265-76. doi:10.2147/OTT.S91941.

17. Bae JM, Shin SH, Kwon HJ, Park SY, Kook MC, Kim YW, et al. ALU and LINE-1 hypomethylations in multistep gastric carcinogenesis and their prognostic implications. Int J Cancer. 2012;131(6):1323-31. doi:10.1002/ijc.27369.

18. Sahnane N, Magnoli F, Bernasconi B, Tibiletti MG, Romualdi C, Pedroni M, et al. Aberrant DNA methylation profiles of inherited and sporadic colorectal cancer. Clin Epigenetics. 2015;7:131. doi:10.1186/s13148-015-0165-2.

19. Harada K, Baba Y, Ishimoto T, Chikamoto A, Kosumi K, Hayashi H, et al. LINE-1 methylation level and patient prognosis in a database of 208 hepatocellular carcinomas. Ann Surg Oncol. 2015;22(4):1280-7. doi:10.1245/s10434-014-4134-3.

20. Baba Y, Murata A, Watanabe M, Baba H. Clinical implications of the LINE-1 methylation levels in patients with gastrointestinal cancer. Surgery today. 2013. doi:10.1007/s00595-013-0763-6.

21. Song YS, Kim Y, Cho NY, Yang HK, Kim WH, Kang GH. Methylation status of long interspersed element-1 in advanced gastric cancer and its prognostic implication. Gastric Cancer. 2016;19(1):98-106. doi:10.1007/s10120-015-0463-6.

22. Dewys WD, Begg C, Lavin PT, Band PR, Bennett JM, Bertino JR, et al. Prognostic effect of weight loss prior to chemotherapy in cancer patients. Eastern Cooperative Oncology Group. Am J Med. 1980;69(4):491-7.

23. Glare $P$, Sinclair $C$, Downing $M$, Stone $P$, Maltoni $M$, Vigano A. Predicting survival in patients with advanced disease. Eur J Cancer. 2008;44(8):1146-56. doi:10.1016/j.ejca.2008.02.030

24. Sengupta A, Banerjee SN, Biswas NM, Jash D, Saha K, Maji A, et al. The incidence of hyponatraemia and its effect on the ECOG performance status among lung cancer patients. J Clin Diagn Res. 2013;7(8):1678-82. doi:10.7860/JCDR/2013/5900.3225.

25. Correa GT, Bandeira GA, Cavalcanti BG, Santos FB, Rodrigues Neto JF, Guimaraes AL, et al. Analysis of ECOG performance status in head and neck squamous cell carcinoma patients: association with sociodemographical 
and clinical factors, and overall survival. Support Care Cancer. 2012;20(11): 2679-85. doi:10.1007/s00520-012-1386-y.

26. Crumley AB, Stuart RC, McKernan M, McDonald AC, McMillan DC. Comparison of an inflammation-based prognostic score (GPS) with performance status (ECOG-ps) in patients receiving palliative chemotherapy for gastroesophageal cancer. J Gastroenterol Hepatol. 2008;23(8 Pt 2):e325-9. doi:10.1111/j.1440-1746.2007.05105.x.

27. Gridelli C, Ardizzoni A, Le Chevalier T, Manegold C, Perrone F, Thatcher N, et al. Treatment of advanced non-small-cell lung cancer patients with ECOG performance status 2: results of an European Experts Panel. Ann Oncol. 2004;15(3):419-26.

28. Forrest LM, McMillan DC, McArdle CS, Angerson WJ, Dunlop DJ. Comparison of an inflammation-based prognostic score (GPS) with performance status (ECOG) in patients receiving platinum-based chemotherapy for inoperable non-small-cell lung cancer. Br J Cancer. 2004;90(9):1704-6. doi:10.1038/sj.bjc. 6601789.

29. Lavin P, Mittelman A, Douglass Jr H, Engstrom P, Klaassen D. Survival and response to chemotherapy for advanced colorectal adenocarcinoma: an Eastern Cooperative Oncology Group report. Cancer. 1980;46(7):1536-43.

30. Crosara Teixeira M, Marques DF, Ferrari AC, Alves MF, Alex AK, Sabbaga J, et al. The effects of palliative chemotherapy in metastatic colorectal cancer patients with an ECOG performance status of 3 and 4 . Clin Colorectal Cancer. 2015;14(1):52-7. doi:10.1016/j.clcc.2014.09.010.

31. Sargent DJ, Kohne CH, Sanoff HK, Bot BM, Seymour MT, de Gramont A, et al. Pooled safety and efficacy analysis examining the effect of performance status on outcomes in nine first-line treatment trials using individual data from patients with metastatic colorectal cancer. J Clin Oncol. 2009;27(12): 1948-55. doi:10.1200/JCO.2008.20.2879.

32. Lu CS, Chang PY, Chen YG, Chen JH, Wu YY, Ho CL. Stage III colon cancer: the individualized strategy of adjuvant chemotherapy for aged under and over 70. PLoS One. 2015;10(9):e0138632. doi:10.1371/journal.pone.0138632.

33. Vergidis J, Gresham G, Lim HJ, Renouf DJ, Kennecke HF, Ruan JY, et al. Impact of weight changes after the diagnosis of stage III colon cancer on survival outcomes. Clin Colorectal Cancer. 2015. doi:10.1016/j.clcc.2015.07.002.

34. Chiappinelli KB, Strissel PL, Desrichard A, Li H, Henke C, Akman B, et al. Inhibiting DNA methylation causes an interferon response in cancer via dsRNA including endogenous retroviruses. Cell. 2015;162(5):974-86. doi:10.1016/j.cell.2015.07.011.

35. Roulois D, Loo Yau H, Singhania R, Wang Y, Danesh A, Shen SY, et al. DNA-demethylating agents target colorectal cancer cells by inducing viral mimicry by endogenous transcripts. Cell. 2015;162(5):961-73. doi:10.1016/j.cell.2015.07.056.

36. MacDonald N, Easson AM, Mazurak VC, Dunn GP, Baracos VE. Understanding and managing cancer cachexia. J Am Coll Surg. 2003:197(1):143-61. doi:10.1016/S1072-7515(03)00382-X.

37. Lou YT, Chen CW, Fan YC, Chang WC, Lu CY, Wu IC, et al. LINE-1 methylation status correlates significantly to post-therapeutic recurrence in stage III colon cancer patients receiving FOLFOX-4 adjuvant chemotherapy. PLoS One. 2014;10(4):e0123973. doi:10.1371/journal.pone.0123973.

38. Stelzner $\mathrm{S}$, Hellmich $\mathrm{G}$, Koch R, Ludwig K. Factors predicting survival in stage IV colorectal carcinoma patients after palliative treatment: a multivariate analysis. J Surg Oncol. 2005;89(4):211-7. doi:10.1002/jso.20196.

39. Janisch L, Mick R, Schilsky RL, Vogelzang NJ, O'Brien S, Kut M, et al. Prognostic factors for survival in patients treated in phase I clinical trials. Cancer. 1994;74(7):1965-73.

40. Weisenberger DJ, Campan M, Long TI, Kim M, Woods C, Fiala E, et al. Analysis of repetitive element DNA methylation by MethyLight. Nucleic Acids Res. 2005;33(21):6823-36. doi:10.1093/nar/gki987.

41. Yang AS, Doshi KD, Choi SW, Mason JB, Mannari RK, Gharybian V, et al. DNA methylation changes after 5-aza-2'-deoxycytidine therapy in patients with leukemia. Cancer Res. 2006;66(10):5495-503. doi:10.1158/0008-5472.CAN-05-2385.

42. Irahara N, Nosho K, Baba Y, Shima K, Lindeman NI, Hazra A, et al. Precision of pyrosequencing assay to measure LINE-1 methylation in colon cancer, normal colonic mucosa, and peripheral blood cells. J Mol Diagn. 2010;12(2): 177-83. doi:10.2353/jmoldx.2010.090106.

43. Tournier B, Chapusot C, Courcet E, Martin L, Lepage C, Faivre J, et al, Why do results conflict regarding the prognostic value of the methylation status in colon cancers? The role of the preservation method. BMC Cancer. 2012;12:12. doi:10.1186/1471-2407-12-12.
44. Song YS, Kim Y, Cho NY, Yang HK, Kim WH, Kang GH. Methylation status of long interspersed element-1 in advanced gastric cancer and its prognostic implication. Gastric Cancer. 2015. doi:10.1007/s10120-015-0463-6.

45. Bae JM, Kim JH, Cho NY, Kim TY, Kang GH. Prognostic implication of the CpG island methylator phenotype in colorectal cancers depends on tumour location. Br J Cancer. 2013;109(4):1004-12. doi:10.1038/bjc.2013.430.

46. Tellez CS, Shen L, Estecio MR, Jelinek J, Gershenwald JE, Issa JP. CpG island methylation profiling in human melanoma cell lines. Melanoma Res. 2009;19(3):146-55.

47. Lee DW, Kim KJ, Han SW, Lee HJ, Rhee YY, Bae JM, et al. KRAS mutation is associated with worse prognosis in stage III or high-risk stage II colon cancer patients treated with adjuvant FOLFOX. Ann Surg Oncol. 2015;22(1): 187-94. doi:10.1245/s10434-014-3826-z.

48. Oken MM, Creech RH, Tormey DC, Horton J, Davis TE, McFadden ET, et al. Toxicity and response criteria of the Eastern Cooperative Oncology Group. Am J Clin Oncol. 1982;5(6):649-55.

49. Ogino S, Kawasaki T, Nosho K, Ohnishi M, Suemoto Y, Kirkner GJ, et al. LINE-1 hypomethylation is inversely associated with microsatellite instability and $\mathrm{CpG}$ island methylator phenotype in colorectal cancer. Int I Cancer. 2008;122(12):2767-73. doi:10.1002/ijc.23470.

50. Rhee YY, Kim MJ, Bae JM, Koh JM, Cho NY, Juhnn YS, et al. Clinical outcomes of patients with microsatellite-unstable colorectal carcinomas depend on L1 methylation level. Ann Surg Oncol. 2012;19(11):3441-8. doi:10.1245/s10434-012-2410-7.

\section{Submit your next manuscript to BioMed Central and we will help you at every step:}

- We accept pre-submission inquiries

- Our selector tool helps you to find the most relevant journal

- We provide round the clock customer support

- Convenient online submission

- Thorough peer review

- Inclusion in PubMed and all major indexing services

- Maximum visibility for your research

Submit your manuscript at www.biomedcentral.com/submit

BioMed Central 